\title{
Subjective and functional results after total gastrectomy: prospective study for longterm comparison of reconstruction procedures
}

\author{
Shinya Adachi, Satoshi Inagawa, Tsuyoshi Enomoto, Eiji Shinozaki, Tatsuya Oda, and Toru Kawamoto \\ Department of Surgery, Institute of Clinical Medicine, University of Tsukuba, 1-1-1 Tennodai, Tsukuba 305-8575, Japan
}

\begin{abstract}
Background. Many reconstruction procedures have been developed in efforts to resolve patients' complaints after total gastrectomy. However, there have been few reports of longterm comparisons between reconstruction procedures, especially with regard to the prevention of duodenal food passage. This study was undertaken to compare the longterm subjective and functional results among Roux-en-Y esophagojejunostomy (R-Y), R-Y with pouch (P-Y), and jejunal interposition with pouch (P-I) after total gastrectomy. Methods. Consecutive patients requiring curative total gastrectomy were enrolled in this prospective study by the envelope method.

Results. Hospital stay was longer following a P-I than an R-Y or a P-Y. Over $50 \%$ of R-Y patients complained of heartburn, and $20 \%$ of $R-Y$ patients showed dumping syndrome throughout the postoperative period, with this rate being significantly different from rates in the other two groups. P-Y patients complained of early satiety in the late postoperative period, while P-I patients complained of early satiety in the early postoperative period. The nutritional index in $\mathrm{P}$-I patients was higher than those in patients with the other two procedures. Gastrointestinal and hepatobiliary dual scintigraphy (GHDS) showed that the rate of bile reflux with an $R-Y$ was relatively high after surgery. Food reflux with a P-Y was increased $(\mathbf{9 . 4} \%$ to $11.1 \%)$, but with a P-I food reflux was decreased at 3 years after surgery $(13.3 \%$ to $9.9 \%)$. Patients with a P-Y had a faster recovery of body, weight in the early postoperative period; however, at 5 years after operation, body weight recovery with a $\mathbf{P}$-I was greatest.

Conclusion. Reconstruction should be performed with pouch formation after total gastrectomy with curative intent.
\end{abstract}

Key words Total gastrectomy $\cdot$ Reconstruction - Nutrition · Bile reflux $\cdot$ Food retention

Offprint requests to: $\mathrm{S}$. Adachi

Received: March 7, 2002 / Accepted: September 26, 2002

\section{Introduction}

The survival rate after total gastrectomy for gastric cancer is improving as a result of earlier diagnosis and more advanced operative techniques [1]. Many reconstruction procedures have been developed in efforts to resolve patients' complaints and to improve the nutritional condition of patients after total gastrectomy. Substitute stomach reconstruction after total gastrectomy seems to be superior to simple esophagojejunostomy from a nutritional-physiologic perspective $[2,3]$. The jejunal pouch reconstruction method is intended to increase the patient's food retention capacity and subsequently to increase body weight recovery. However, this time-consuming manual reconstruction of a pouch results in increased operative stress, morbidity, and mortality. Thiede et al. [4] have reported that the exclusive use of autosuturing surgical devices during reconstruction results in intraoperative time saving. Since their report, several efforts to use the jejunal pouch in reconstruction have been made [5,6]. In Japan, Rouxen-Y esophagojejunostomy (R-Y) is the most commonly used mode of reconstruction following total gastrectomy. In order to resolve the major complaints after an R-Y following total gastrectomy (e.g., heartburn, reduction of food capacity, and body weight loss), starting in 1991, we [7] have performed jejunal pouch reconstructions following total gastrectomy for patients with gastric malignancy. Since 1987, several randomized prospective clinical trials comparing various methods of intestinal reconstruction following total gastrectomy have been reported [8-13]. However, the longterm impact of different reconstructive procedures, especially on duodenal food passage, has not been investigated. This study was undertaken to compare the longterm postoperative subjective and functional results of three reconstruction procedures, R-Y, Roux-en-Y with pouch (P-Y), and jejunal interposition with pouch (P-I), after total gastrectomy. We previously reported vitamin B12 
$\left(\mathrm{VB}_{12}\right)$ deficiency symptoms causing lower quality of life (QOL) after total gastrectomy [14], and showed that enteral $\mathrm{VB}_{12}$ administration corrected the hypovitaminemia irrespective of the reconstruction procedures used $[14,15]$. All of the patients enrolled in this study received enteral and/or parenteral $\mathrm{VB}_{12}$ supplements.

\section{Patients and methods}

\section{Patients}

Consecutive patients with gastric malignancies who were expected to have a curative total gastric resection were enrolled from 1995 to 1996 . They were randomly divided into three groups: R-Y, P-Y, or P-I, by a sealed envelope method. If a recurrent lesion became apparent or follow-up was impossible within 2 years after operation, a new patient was enrolled in the same reconstruction group. Of the 34 consecutive patients assigned to the three different reconstruction groups, 4 patients relapsed, while 30 patients (28 cancers, 2 mucosaassociated lymphoid tissue [MALT] lymphomas) were able to be followed over the next 5 years.

\section{Reconstructions}

Following curative total gastrectomy and systematic lymphadenectomy, the distance of the esophagojejunostomy to the jejunojejunostomy was $40 \mathrm{~cm}$ for the R-Y. To shape the pouch, doubled loops of the jejunum were stapled together in two or three steps with a double stapling instrument and were cut to form the substitute stomach. No manual sutures were added for hemostasis. The pouch was $20 \mathrm{~cm}$ long, with a $20-\mathrm{cm}$ efferent jejunal loop with the P-Y, and a 10-cm jejunal loop with the P-I (Fig. 1).
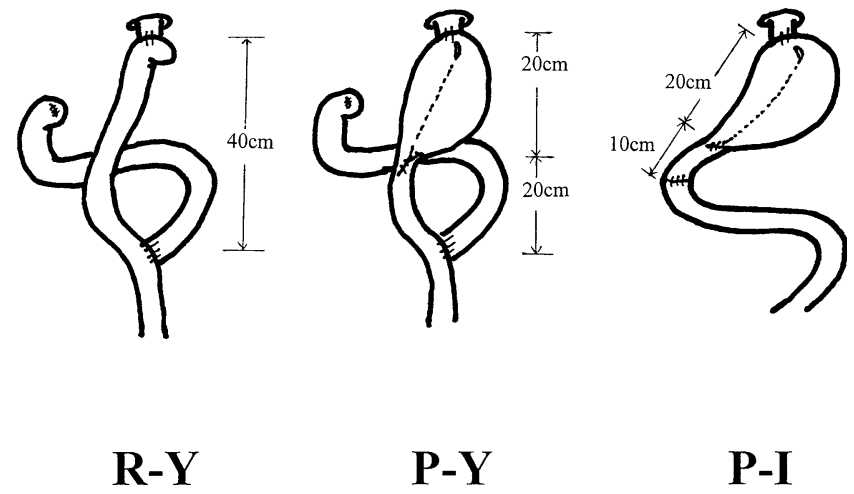

Fig. 1. Illustrated reconstruction procedures. $R-Y$, Roux-en-Y esophagojejunostomy; $P-Y$, Roux-en-Y with pouch; $P-I$, jejunal interposition with pouch

\section{Evaluations}

We compared the three groups regarding their perioperative condition, complications, and hospital stay. As the endpoint of the evaluation, the following were tracked for 5 years: patient complaints, changes in the body weight, and nutritional evaluations. Onodera's index: $10 \times$ serum albumin $(\mathrm{g} / \mathrm{dl})+0.005 \times$ lymphocyte count, and the nutrition risk index (NRI): $1.519 \times$ serum albumin $(\mathrm{g} / \mathrm{l})+0.417 \times$ (current weight/usual weight $) \times 100$ were used as the nutritional evaluation [16]. Gastrointestinal and hepatobiliary dual scintigraphy (GHDS) was performed at 1 and 3 years after gastrectomy for all patients, with their informed consent [17].

\section{Statistical analysis}

The Kruskal-Wallis test, with the Mann-Whitney $U$ test with Bonferroni correction, and repeated-measure analysis of variance (ANOVA) with Fisher's protected least significant difference (PLSD) were used. Differences were considered significant at $P<0.05$.

\section{Results}

The R-Y group included 8 men and 2 women, the P-Y group, 6 men and 4 women, and the P-I group, 9 men and 1 woman. The median age of the patients undergoing R-Y was 59.0 years (range, 43 to 74 years); P-Y, 57.5 years (range, 48-75 years); and P-I, 53.0 years (range, 38-72 years). According to the Japanese classification, 11 patients were classified as stage Ia, 5 patients as stage $\mathrm{Ib}, 8$ patients as stage II, and 6 patients as stage IIIa [18]. There were no significant differences in the patients' background data among the three groups. According to the curative potential classification, 22 patients had Resection A and 8 patients had Resection B [18] (Table 1). Seventeen patients received a splenectomy to improve their cure rate.

Operating time, blood loss, and morbidity rates showed no significant differences among the groups. However, the length of hospital stay after surgery in the P-I group was longer than those for the other two groups (Table 2).

In regard to patients' symptoms, R-Y patients complained of heartburn throughout the postoperative period. This rate was significantly higher than that of the other two groups. P-Y patients complained of early satiety in the late postoperative period. Conversely, P-I patients complained of early satiety in the early postoperative period. This sensation was felt within $30 \mathrm{~min}$ after beginning a meal. Rates for dumping syndrome were significantly lower in both pouch groups than in 


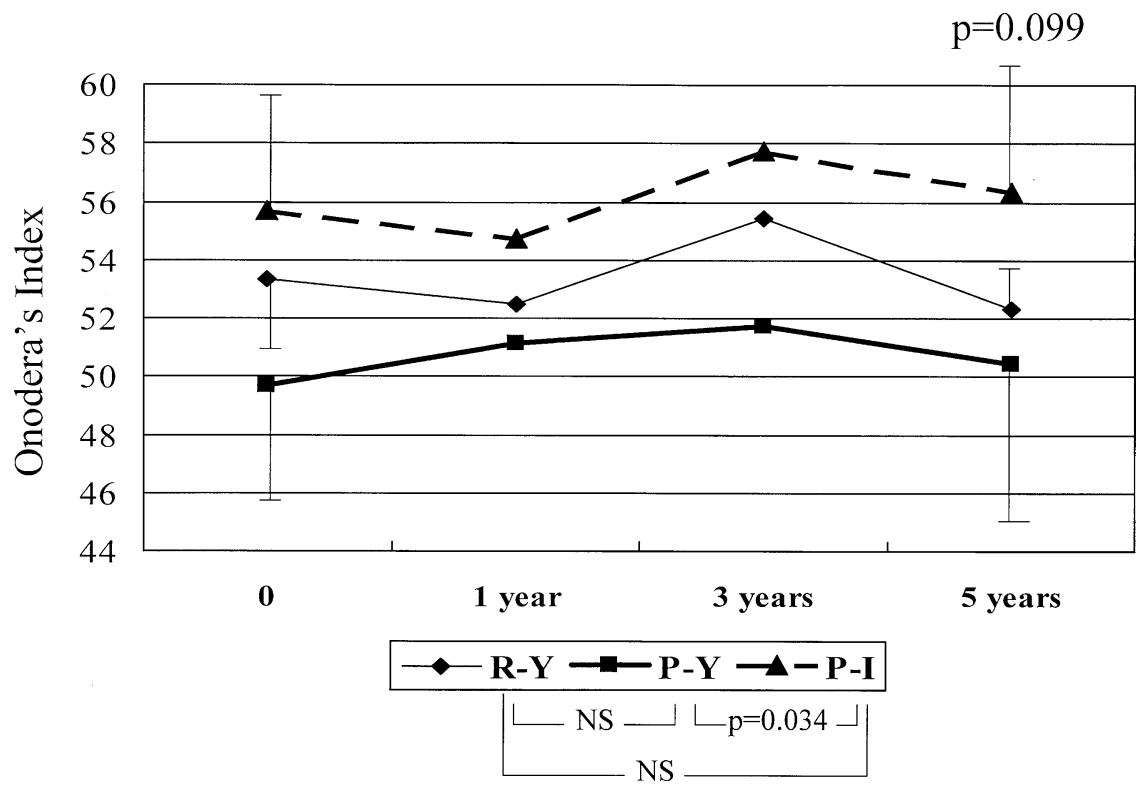

Fig. 2. Onodera's index: $10 \times$ serum albumin $(\mathrm{g} / \mathrm{dl})+0.005 \times$ lymphocyte count repeated-measure analysis of variance (ANOVA) with Fisher's protected least significant difference (PLSD). NS, Not significant

Table 1. Patients' backgrounds

\begin{tabular}{lccc}
\hline & R-Y & P-Y & P-I \\
\hline Age (years) & $59.0($ Range, $43-74)$ & $57.5($ Range, $48-75)$ & 53.0 (Range, 38-72) \\
M:F & $8: 2$ & $6: 4$ & $9: 1$ \\
Stage & & & 3 \\
Ia & 4 & 4 & 2 \\
Ib & 2 & 1 & 3 \\
II & 2 & 3 & 2 \\
IIIa & 2 & 2 & $4: 6$ \\
D1:D2 & $4: 6$ & $5: 5$ & 7 \\
Resection A & 8 & 7 & 3 \\
Resection B $^{\text {a }}$ & 2 & 3 & \\
\hline
\end{tabular}

Kruskal-Wallis test, Not significent (NS)

R-Y, Roux-en-Y esophagojejunostomy; P-Y, R-Y with pouch; P-I, jejunal interposition with pouch

${ }^{a}$ According to Japanese Classification of Gastric Carcinoma [18]

Table 2. Perioperative status

\begin{tabular}{|c|c|c|c|}
\hline & $\mathrm{R}-\mathrm{Y}$ & $\mathrm{P}-\mathrm{Y}$ & P-I \\
\hline Operating time (min) & $272 \pm 60$ & $259 \pm 43$ & $312 \pm 69$ \\
\hline Blood loss $(\mathrm{ml})$ & $552 \pm 255$ & $678 \pm 409$ & $719 \pm 321$ \\
\hline Pneumonia & 1 & 1 & 2 \\
\hline Leakage & 0 & 0 & 1 \\
\hline Peritoneal abscess & 1 & 1 & 2 \\
\hline \multirow[t]{3}{*}{ Hospital stay (days) ${ }^{\mathrm{a}}$} & $33 \pm 8$ & $27 \pm 6$ & $42 \pm 13$ \\
\hline & & \multicolumn{2}{|c|}{$P=0.0137$} \\
\hline & & $P=0.048$ & \\
\hline
\end{tabular}

${ }^{\mathrm{a}}$ Kruskal-Wallis test; $P=0.025$

the R-Y group throughout the postoperative period (Table 3). NRI and Onodera's index showed differences approaching statistical significance among the three groups $(P=0.102 ; P=0.099)$. The Onodera's index of a P-I was higher than that of a P-Y (Fig. 2), and the NRI score of a P-I was higher than that of an R-Y (Fig. 3).

GHDS showed that bile reflux with an R-Y was relatively high at 1 and 3 years after operation. Bile reflux with a P-I gradually decreased. Food reflux with a P-Y was increased, but with a P-I, food reflux was decreased at 3 years after the surgery. The 1-h food retention rates in the pouch were similar in both the P-Y and P-I groups. However, five of eight patients with a P-Y had an increase in their 1-h food retention rate at 3 years after operation, while four of six patients with a P-I had a decrease in their 1 -h food retention rate at 3 years after surgery $(P=0.296$; Table 4$)$.

Changes in body weights showed differences approaching statistical significance among the three groups $(P=0.089)$. The body weight recovery was significantly worse with an R-Y (Fig. 4). Body weight at 5 years after operation as a percentage of preoperative 
Table 3. Symptoms (\%)

\begin{tabular}{|c|c|c|c|c|c|c|c|c|}
\hline \multicolumn{3}{|c|}{ R-Y } & \multicolumn{3}{|c|}{ P-Y } & \multicolumn{3}{|c|}{ P-I } \\
\hline $1-$, & 3-, & 5-Year & $1-$, & $3-$ & 5-Year & 1 , & 3-, & 5-Year \\
\hline
\end{tabular}

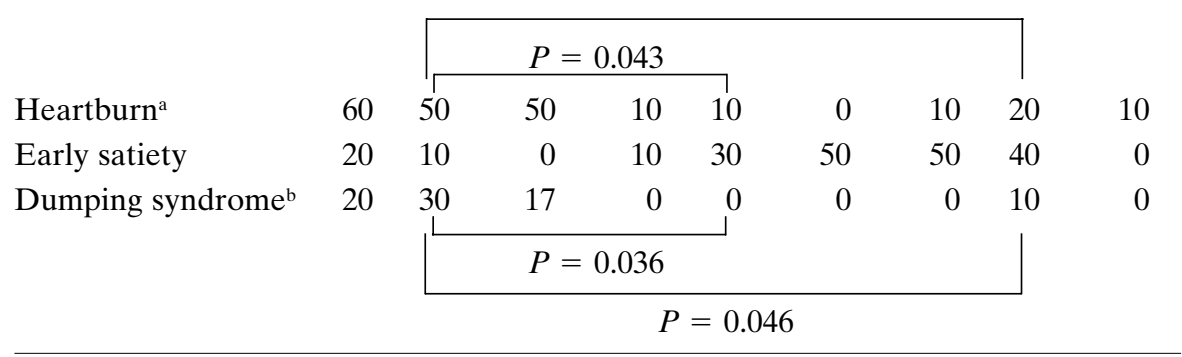

${ }^{\mathrm{a}}$ Kruskal-Wallis test; $P=0.037 ;{ }^{\mathrm{b}}$ Kruskal-Wallis test; $P=0.034$

Table 4. Gastrointestinal and hepatobiliary dual scintigraphy (\%)

\begin{tabular}{|c|c|c|c|c|c|c|}
\hline & \multicolumn{2}{|c|}{$\mathrm{R}-\mathrm{Y}$} & \multicolumn{2}{|c|}{ P-Y } & \multicolumn{2}{|c|}{ P-I } \\
\hline & $1-$ & 3-Year & $1-$ & 3-Year & $1-$ & 3-Year \\
\hline Bile reflux $(n)$ & 86 & 67 & 20 & 0 & 50 & 17 \\
\hline Food reflux (counts) & 9.9 & 1.2 & 9.4 & 11.1 & 13.3 & 9.9 \\
\hline 1-h retention (counts) & & & 73 & 67 & 68 & 63 \\
\hline$[1$ year $>3$ years $(n / n)]$ & & & \multicolumn{2}{|c|}{$3 / 8$} & \multicolumn{2}{|c|}{$4 / 6$} \\
\hline
\end{tabular}

Kruskal-Wallis test, NS

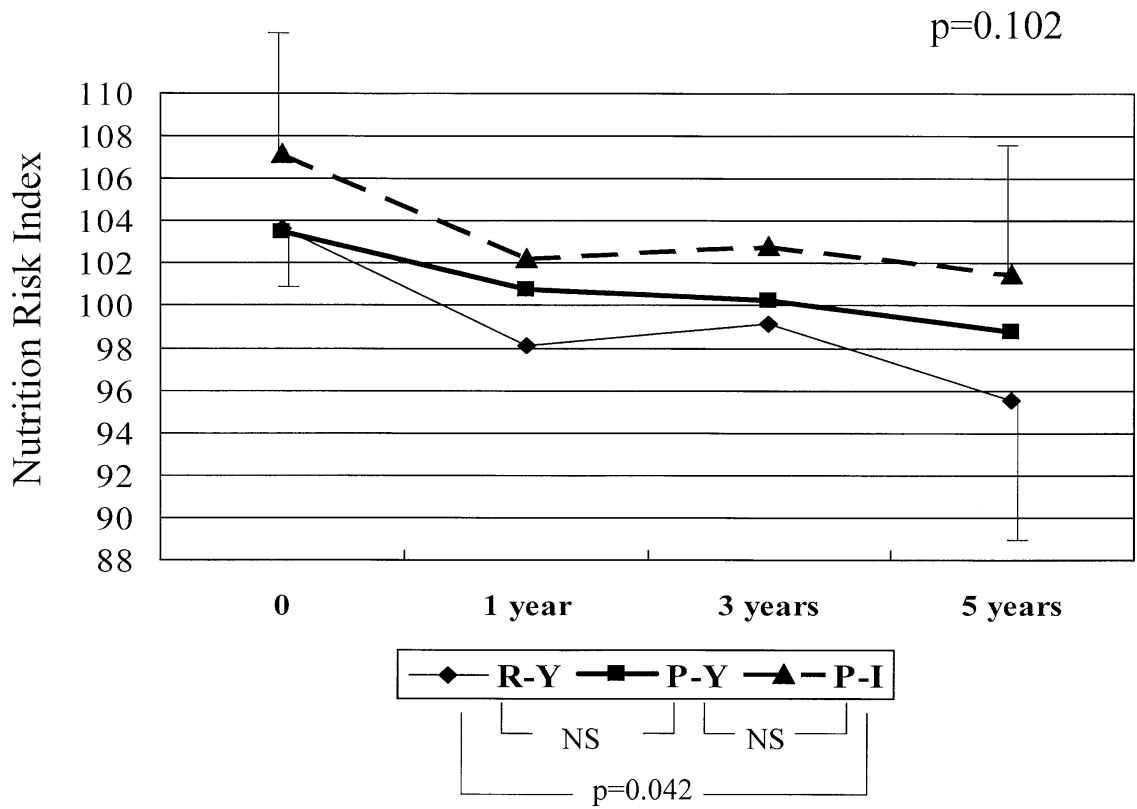

Fig. 3. Nutrition risk index: $1.519 \times$ serum albumin $(\mathrm{g} / \mathrm{l})+0.417 \times$ (current weight/ usual weight $) \times 100$; repeatedmeasure ANOVA with Fisher's PLSD weight was $86.9 \%$ with an R-Y, $94.9 \%$ with a P-Y, and $95.1 \%$ with a P-I.

\section{Discussion}

Assessment of postoperative QOL depends on patients' perceptions. Therefore, in order to evaluate and com- pare reconstruction procedures in regard to postoperative QOL, it is necessary for outpatients to receive the best possible level of supportive care. In addition, objective parameters of QOL are also available. Total gastrectomy results in some reversible loss of function, as well as some irreversible loss of function. Vitamin $B_{12}$ $\left(\mathrm{VB}_{12}\right)$ deficiency can develop as early as 1 year after total gastrectomy prior to the onset of anemia $[19,20]$. 


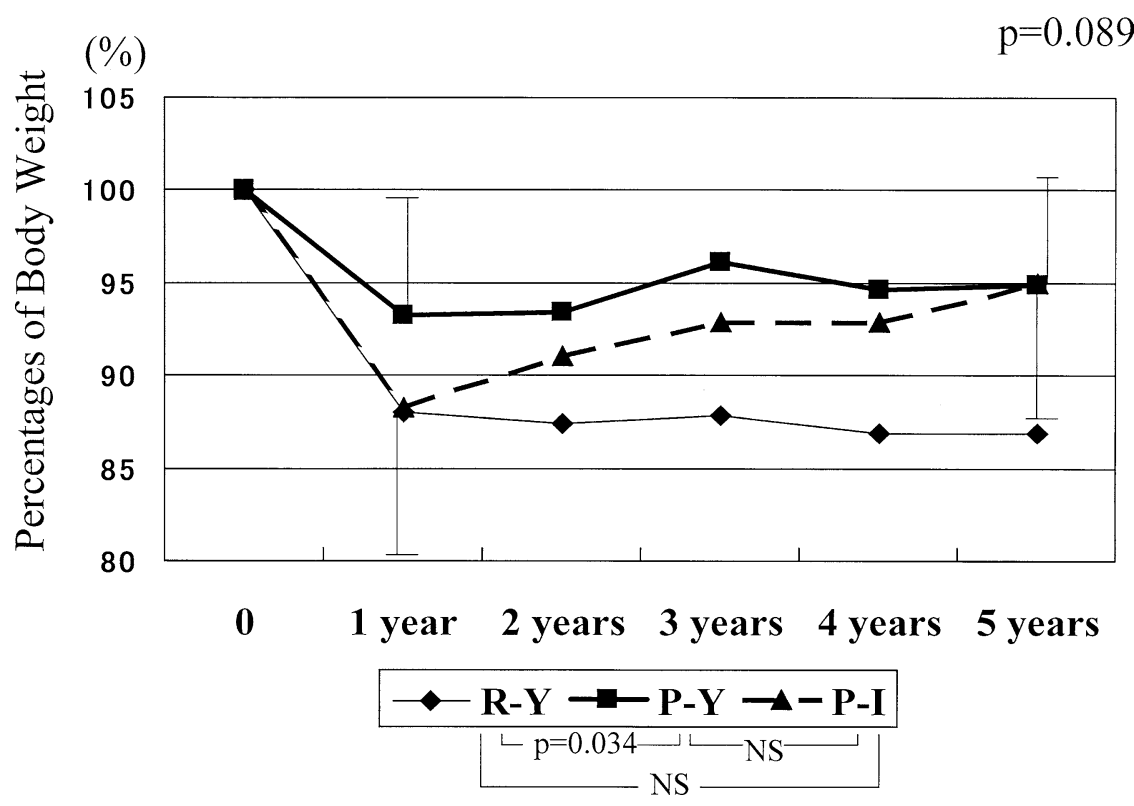

Fig. 4. Body weights of the patients after operation; repeated-measure ANOVA with Fisher's PLSD
The treatment of pernicious anemia due to $\mathrm{VB}_{12}$ deficiency has been thought to require parenteral $\mathrm{VB}_{12}$ administration [21]. However, enteral $\mathrm{VB}_{12}$ treatment increases the serum $\mathrm{VB}_{12}$ concentration, while symptoms related to $\mathrm{VB}_{12}$ deficiency rapidly resolve [15]. Patients who were registered for this research received oral $\mathrm{VB}_{12}$ supplements of the ideal quantity for vitamin deficiency [15]. This resolved complaints according to $\mathrm{VB}_{12}$ deficiency, while the remaining complaints, such as heartburn, early satiety, and dumping syndrome, seemed to be dependent on the reconstruction procedures.

The following results approached or reached statistically significant differences: heartburn and dumping syndrome in $\mathrm{R}-\mathrm{Y}$, and hospitalization and nutritional index in P-I were higher; also, there was earlier body weight recovery in $\mathrm{P}-\mathrm{Y}$ versus $\mathrm{R}-\mathrm{Y}$, with lowest recovery in R-Y. There were no significant differences in the operating times, but operations that exceeded $5 \mathrm{~h}$, were performed in three patients with an R-Y, two with a P$\mathrm{Y}$, and six with a P-I. Although the comparisons did not distinguish the time for resection from the time for the reconstruction, differences in the time taken for resection by each operator seemed to lead to the results above. The postoperative hospital stay is influenced by whether or not a patient has complications. The numbers of patients who were hospitalized for over 50 days were: one with an R-Y, three with a P-I, and none with a P-Y. Three patients had an intraabdominal abscess, and one patient had leakage at the anastomosis site.

Patients who underwent an R-Y had higher rates of heartburn and dumping syndrome, esophageal bile reflux on GHDS, and the lowest NRI, and their weight decreased significantly. Patients who underwent a P-Y had the lowest bile reflux rate, but complained of early satiety leading to passage disturbance at later postoperative stages. Their body weight increase was fast in the early postoperative phase. A jejunal pouch can also store a meal, but there is a danger of disturbing meal passage, leading to early satiety and esophagitis due to retained food $[22,23]$. Patients who underwent a P-I had a higher bile reflux rate during the early postoperative course, but this rate had decreased 3 years after surgery on GHDS. The body weight of patients with a P-I gradually increased, and was nearest to the preoperative body weight at 5 years after total gastrectomy among the three groups. The 1-h food retention rates in the pouch were similar in both the P-Y and P-I groups. However, with increased postoperative time, four of six patients with a P-I had a decrease in their 1-h food retention rate, while five of eight patients with a P-Y had an increase in their 1-h food retention rate. With a $\mathrm{P}-\mathrm{I}$, a decrease in the 1-h food retention rate in the late phase brought about a good result. The benefit of preserving the duodenal food passage was not significant. However, with a P-I, the highest NRI score and Onodera's index and good body weight recovery from 1 to 5 years after surgery (if a more longterm observation is carried out) show that a nutritionally good result may be brought about. In the review article of Espat and Karpeh [24], the lack of standardization and the variable outcomes fail to support any single operation as the preferred method of reconstruction following total gastrectomy. In the present study, jejunal pouch reconstruction was superior from the nutritional aspect. Additionally, the relatively high frequency of gallstones 
after gastrectomy is well known. The preservation of the duodenal passage is advantageous for the patient with gallstones by allowing interventional treatment such as endoscopic retrograde cholangiopancreatography, biliary drainage, and endoscopic sphincterotomy, even for the patient with cholangiopancreatic malignancy.

The pouch group was superior to an R-Y concerning QOL and functional results in the postoperative period. Reconstruction should be performed with pouch formation after total gastrectomy with curative intent. With a $\mathrm{P}-\mathrm{Y}$, the superiority was remarked in the early postoperative period, while it was less marked in the late stage. However, with a P-I, functional results were improved over the 3-year postoperative period. From the above results, it seems that one to ought to choose a P-I for patients in whom longterm survival is expected (Resection $\mathrm{A}$ ), and a $\mathrm{P}-\mathrm{Y}$ in those in whom longterm survival is not very much expected (Resection B).

Acknowledgments This study was partly supported by the University of Tsukuba Research Project.

\section{References}

1. Fujii M, Sasaki J, Nakajima T. State of the art in the treatment of gastric cancer: from the 71st Japanese Gastric Cancer Congress. Gastric Cancer 1999;2:151-7.

2. Hunt CJ. Construction of food pouch from segment of jejunum as a substitute for stomach in total gastrectomy. Arch Surg 1952; 64:601-8.

3. Lawrence W Jr. Reservoir construction after total gastrectomy. Ann Surg 1962;155:191-8.

4. Thiede A, Fuchs KH, Hamelman H. Pouch and Roux-en-Y reconstruction after gastrectomy. Arch Surg 1987;122:83742.

5. Fujiwara Y, Kusunoki M, Nakagawa K, Tanaka T, Hatada T, Yamamura T. Evaluation of J-pouch reconstruction after total gastrectomy: rho-double tract vs J-pouch double tract. Dig Surg 2000;17:475-82.

6. Gioffre'Florio MA, Bartolotta M, Miceli JC, Giacobbe G, Saitta FP, Paparo MT, Micali B. Simple versus double jejunal pouch for reconstruction after total gastrectomy. Am J Surg 2000;180:248.

7. Adachi S, Fukao K, Kawashima T, Ozaki A. Usefulness of jejunal pouch reconstruction after total gastric resection. In: Nishi M, Sugano H, Takahashi T, editors. First International Gastric Cancer Congress. Bologna, Italy: Monduzzi Editore; 1995. p 132731.
8. Troidl H, Kusche J, Vestweber KH, Eypasch E, Maul U. Pouch versus esophago-jejunostomy after total gastrectomy: a randomized clinical trial. World J Surg 1987;11:699-712.

9. Schmitz R, Moser KH, Treckmann J. Quality of life after programmed jejunum interposition with and without pouch. A prospective study of stomach cancer patients on the reservoir as a reconstruction principle after total gastrectomy. Chirurg 1994;65: 326-32.

10. Nakane Y, Okumura S, Akehira K, Okamura S, Boku T, Okusa $\mathrm{T}$, et al. Jejunal pouch reconstruction after total gastrectomy for cancer. A randomized controlled trial. Ann Surg 1995;222:27-35.

11. Fuchs KH, Thiede A, Engemann R, Deltz E, Stremme O, Hamelmann H. Reconstruction of the food passage after total gastrectomy: randomized trial. World J Surg 1995;19:698-705.

12. Schwarz A, Buchler M, Usinger K, Rieger H, Glasbrenner B, Friess $\mathrm{H}$, et al. Importance of the duodenal passage and pouch volume after total gastrectomy and reconstruction with the Ulm pouch: prospective randomized clinical study. World J Surg 1996; 20:60-6.

13. Bozzetti F, Bonfanti G, Castellani R, Maffioli L, Rubino A, Diazzi $\mathrm{G}$, et al. Comparing reconstruction with Roux-en-Y to a pouch following total gastrectomy. J Am Coll Surg 1996;183:243-8.

14. Adachi S, Kawamoto T, Inagawa S, Enomoto T, Shinozaki E, Oda T, Fukao K. Vitamin B12 concentration after total gastrectomy and enteral supplement. In: Brennan MF, Karpeh MS, editors. Fourth International Gastric Cancer Congress. Bologna, Italy: Monduzzi Editore; 2001. p 645-8.

15. Adachi S, Kawamoto T, Otsuka M, Todoroki T, Fukao K. Enteral vitamin B12 supplements reverse postgastrectomy B12 deficiency. Ann Surg 2000;232:199-201.

16. The Veterans Affairs Total Parenteral Nutrition Cooperative Study Group. Perioperative total parenteral nutrition in surgical patients. N Engl J Med 1991;325:525-2.

17. Adachi S, Takeda T, Fukao K. Evaluation of esophageal bile reflux after total gastrectomy by gastrointestinal and hepatobiliary dual scintigraphy. Surg Today 1999;29:301-6.

18. Japanese Gastric Cancer Association. Japanese classification of gastric carcinoma. 2nd English E. Gastric Cancer 1998;1:10-24.

19. Haryu E. Metabolic problems after gastric surgery. Int Surg 1990; 75:27-35.

20. Bae JM, Park JW, Yang HK, Kim JP. Nutritional status of gastric cancer patients after total gastrectomy. World J Surg 1998;22:25461.

21. Okuda K. Discovery of vitamin B12 in the liver and its absorption factor in the stomach: a historical review. J Gastroenterol Hepatol 1999;14:301-8.

22. Iivonen MK, Koshinen MO, Ikonen TJ, Matikainen MJ, Jivonen MK. Emptying of the jejunal pouch and Roux-en-Y limb after total gastrectomy - a randomized, prospective study. Eur J Surg 1999;165:742-7.

23. Iivonen MK, Mattila JJ, Nordback IH, Matikainen MJ. Longterm follow-up of patients with jejunal pouch reconstruction after total gastrectomy. A randomized prospective study. Scand J Gastroenterol 2000;35:679-85.

24. Espat NJ, Karpeh M. Reconstruction following total gastrectomy: a review and summary of the randomized prospective clinical trials. Surg Oncol 1999;7:65-9. 